\title{
Special Section Guest Editorial: Frontiers of Optical Coatings
}

\author{
Xinbin Cheng, ${ }^{a}$ Sven Schroder, ${ }^{b}$ Christopher Stolz, ${ }^{c}$ and Xu Liu ${ }^{d}$ \\ ${ }^{a}$ Tongji University, Institute of Precision Optical Engineering, \\ School of Physics Science and Engineering, Shanghai, China \\ ${ }^{b}$ Fraunhofer Institute for Applied Optics and Precision Engineering IOF, Jena, Germany \\ ${ }^{\mathrm{c}}$ Lawrence Livermore National Laboratory, Livermore, California, United States \\ ${ }^{\mathrm{d}}$ Zhejiang University, Department of Optical Engineering, Hangzhou, China
}

Optical coatings is one of the core technologies in modern optical fields. Recent progress in the field of materials, design theory, instruments, and techniques of optical coatings opens up a new avenue for developing advanced thin film optical filters, low-loss coatings, high power laser coatings and novel functional optical coatings that are capable of solving numerous complex problems in areas such as biomedical, laser systems, security, remote sensing, astronomy, and aerospace.

The Frontiers of Optical Coatings (FOC) conference is the top-level conference about optical coatings held in Asia and also one of the largest and highest level academic conferences around the world. The last four FOC conferences have attracted scientists from around the world to present the latest scientific and industry developments of the optical coating domain and achieved complete success.

Considering this rapid progress of optical coatings and the high-impact of the FOC conference, Optical Engineering intended to publish a special section of selected papers from Frontiers of Optical Coatings 2020. Unfortunately, because of the COVID-19 pandemic and the resulting slowdown in research activity, the conference was canceled. However, we still received several submissions, and after rigorous review, Optical Engineering has published the special section.

The published special section consists of four papers that related to recent advances in optical coating materials. The challenge of a high-performance $\mathrm{HfO}_{2}$ film is to simultaneously obtain an amorphous morphology with low scattering and a stoichiometric structure with low absorption. Niu et al. present a detailed study of the crystallization, surface topography, and absorption evolution in $\mathrm{HfO}_{2} / \mathrm{SiO}_{2}$ nanolaminates as a function of $\mathrm{HfO}_{2}$ sublayer thickness and thermal annealing temperature. A 1064-nm $\mathrm{HfO}_{2} / \mathrm{SiO}_{2}$ nanolaminate- $\mathrm{SiO}_{2}$ high reflector with low absorption and a smooth surface has been prepared, which demonstrates the great potential of $\mathrm{HfO}_{2} / \mathrm{SiO}_{2}$ nanolaminates for considerably improving optical loss.

Bai et al. systematically studied the correlation between the process parameters of auxiliary ion source and the $\mathrm{SiO}_{2}$ film stress using dual-ion-sputtering deposition. The experimental result shows that high-energy oxygen ion-assisted deposition can significantly reduce the film stress and the assisted ion beam voltage plays an important role in controlling it. Ultra-low stress high reflective coating was produced by dual-ion-beam sputtering deposition, and can ensure sufficiently high optical quality to keep it reliable and stable in high-precision laser systems

Jiang et al. introduced a high-temperature infrared spectrum measuring device and studied the stability of the $\mathrm{SiO}_{2}$ films deposited with different techniques. It demonstrates that $\mathrm{IBS}-\mathrm{SiO}_{2}$ and $\mathrm{IAD}-\mathrm{SiO}_{2}$ films are more stable than $\mathrm{EB}-\mathrm{SiO}_{2}$ film at the operating temperature higher than $300^{\circ} \mathrm{C}$. Moreover, the complex dielectric functions fitted from FTIR transmittance spectra show that the structure of the $\mathrm{Si}-\mathrm{O}-\mathrm{Si}$ network in $\mathrm{IBS}_{-} \mathrm{SiO}_{2}$ films is the most stable structure.

The reflectivity of $\mathrm{Al} / \mathrm{LiF}$ mirrors decrease rapidly in an environment with a high relative humidity $(\mathrm{RH})$ due to the hygroscopicity of LiF. Li et al. investigated the effect of the humidity to $\mathrm{Al}$ mirrors protected with $\mathrm{LiF}$ and enhanced $\mathrm{MgF}_{2}$ within the $\mathrm{FUV}$ spectrum. It illustrates that the reflectivity remains stable when the environment is $20 \% \mathrm{RH}$, and the reflectivity decreases

(C) 2021 Society of Photo-Optical Instrumentation Engineers (SPIE) 
and the roughness increases over time in the case of high RH. Furthermore, the degradation of reflectivity can be attributed to the presence of oxygen in environments with a high $\mathrm{RH}$.

The guest editors would like to thank the contributors, reviewers, and the Optical Engineering editorial staff for their great work and help during the preparation of this special section. 